\title{
MUJERES Y ESTUDIOS TÉCNICOS, ¿CONCEPTOS COMPATIBLES? TENTATIVA DE UNA COMPARACIÓN INTERCULTURAL ENTRE ALEMANIA Y BRASIL ${ }^{14}$
}

\author{
Irmtraud Munder ${ }^{1}$, Karin Töpsch², \\ Iris Tinsel ${ }^{2}$, Marilia Gomes de Carvalho ${ }^{3}$, \\ Kazuo Hatakeyama ${ }^{3}$
}

\section{INTRODUCCIÓN}

La relación de género en general y la division de trabajo entre mujeres y hombres en particular se está cada vez más cuestionada en un gran número de países así como en varias áreas de la vida. Influencias diferentes han desencadenado esta evolución, abarcando desde la discusión de los derechos humanos hasta la disponibilidad de recursos humanos, de los cuales las mujeres representan la mitad.

La estadística de las Naciones Unidas acerca del Gender Empowerment Measures ${ }^{4}$ señala que los gobiernos reaccionaban de maneras distintas, llegando a medidas tan amplias como el concepto del Gender Mainstreaming de la Union Europea sobre una simple retórica política sin ninguna acción hasta el ignorar el tema. Observando no sólo al Gender Empowerment Measures, sino también a la selección de profesiones de mujeres y la proporción de mujeres en varias disciplinas académicas notamos desarollos interesantes con diferentes paises del mundo: En muchos países, por ejemplo Alemania y México ${ }^{5}$, encontramos un número mayor de mujeres en áreas profesionales como la medicina, life sciences, humanidades, mas un número menor en las ciencias exactas o ingenierías. En el caso de México, Florentina Preciado Cortés explica esta observación diciendo que las profesiones preferidas para las mujeres aprecian de alguna manera como

1 Ponencia para el V Congreso Iberoaméricano "Ciencia, Tecnología y Género" del 16 al 20 febrero 2004 en México D.F. Ponencia oral.

2 Centro de Competencia de Género en Tecnología,

Escuela Técnica Superior de Furtwangen,

Robert-Gerwig-Platz 1,

78120 Furtwangen/ Alemania

munder@fh-furtwangen.de

3 Grupo de Estudos e Pesquisas em Relaçōes de Género e Tecnologia

Programa de Pós-graduação em Tecnologia

Centro Federal de Educação Tecnologica do Paraná en Brasil

Av. Sete de Septembro, 3165

80230-910 Curitiba-Pr, Brasil

carvalho@ppgte.cefepr.br

4 http://www.undp.org/hdr2002/presskit/HDR\%20PR_GEM.pdf

5 Rivera Gómez, Elva: Género, educación y Universidad. Un acercamiento a la historigrafia en México, Ponencia presentada en el Congreso Centroamericano de Historia, Panamá, julio 2002 p. 6 
extensión de lo doméstico, con cierta compatibilidad con el papel de esposa y de madre abnegada ${ }^{6}$. En algunos países observamos un cambio rápido que está equilibrando la distribución de áreas de estudio por sexo. Por frustración de los simpatizantes de cuotas o de una política de regularización no se logra probar una relación directa entre los Gender Empowerment Measures de los gobiernos y el dinamismo de distribución de áreas de estudio por sexo. Por ejemplo el porcentaje de mujeres en las ciencias exactas y en ingeníeras es uno de los más altos en Irán y en Turkía ${ }^{7}$, que son países que no cuentan con programas que promueven la participación de las mujeres. En Alemania y Noruega solamente notamos mínimos cambios en la proporción por sexo pese a los intensos esfuerzos de sus gobiernos. Finalmente, se dice que en el caso de Brasil las medidas políticas y el proceso de contrarrestar la matrícula masculina y femenina en estudios tecnológicos se desarrollan hacía la misma dirección ${ }^{8}$.

Evidentemente los caminos seguidos por los países no dependen sólo del grado de industrialización o de modernización, sino también de la cultura en un sentido amplio. Esta aportación se funda preponderantamente en investigaciones a largo plazo para la relación de género y estudios tecnológicos. Éstas se diseñaron y se llevaron a cabo independientemente en dos instituciones de educación superior semejantes, ambas con una orientación tecnológica: la Furtwangen Universidad en Alemania (FHF) y el Centro Federal de Educação Tecnológica do Paraná (CEFET-PR) en Brasil. A partir de la comparación de los resultados de estas dos investigaciónes, vamos a determinar la influencia, que tienen las diferentes culturas en la selección de áreas de estudios y profesiones académicas por sexo.

Por encima de la influencia de la cultura cabe preguntarnos si la proporción actual de los sexos en las especialidades de las universidades está en consenso con la idéa de la democracia de género, más concreto: si el contenido de las clases, la didáctica y la atmósfera en las facultades fomenta suficientemente todos y todas en desarrollar sus personalidades individuales y unicas.

\section{LA VISTA SOBRE GÉNERO, CIENCIA Y TECNOLOGÍA}

Al principio vamos a dar una idea géneral de la situación de los dos países, Alemania y Brasil, en una escala nacional. Ellas forman las condiciones marco por la situación en las escuelas tecnológicas superiores, que vamos a describir y analizar en detalles en los parafos posteriores. En ambos países la elección del área del estudio lleva un aspecto especifico por el sexo, las chicas prefieren otras especialidades que los chicos. Esta actitud no merecería mencionarse, si las diferentes especialidades no llevaran a oportunidades de carrera diversas, que reforzan la desigualdad género-especifica como la

6 Preciado Cortés, Florentina: Lo público y lo privato en la educación femenina del occidente mexicano. En: GénEros, Vol 4, No. 11, 30-37, 2/1997 citado para Rivera Gómez, Elva ibid.

http:/www.oed.org

8 Gomes de Carvalho, Marilia: Women in Science and Technology : Changes and Transformations. 7th International Conference on Technology Policy and Innovation, Monterrey, 2003 
diferencia de ingreso, estatus, o la participación politica entre hombres y mujeres y infringen con ello contra los principios de gender mainstreaming. A parte en Alemania, un país con una tasa de natalidad más pequeña del mundo, ya se preocupan organisaciones como la Asociación de Ingeneros Alemanos $(\mathrm{VDI})^{9}$ o la Asociación de la Industria Alemana (BDI) por la carencia de la nueva generación de las/los ingenieres. Éllas exigen acciones para tratar de ganarse a mas mujeres para profesiones técnicas con el fin de aliviar la escasez de los ingenieros. La industria en Brasil, un país con una población joven, no tiene este interés intrinsico por las mujeres.

\subsection{La vista nacional en Brasil}

Los datos aquí presentados son del Consejo Nacional de Desarrollo Científico y Tecnológico, del Ministerio de Ciencia y Tecnologia, do año de 2002 (CNPq) del govierno brasileño y muestran la participación de investigadoras e investigadores en las diferentes áreas del conocimiento por edad y titulación.

Se puede decir que la participación de las mujeres en ciencia y tecnología tuvo inicio efectivamente después del movimiento femenino de la década de 60 y que crece año a año. Eso proceso está difundiendose también para otras dimensiones de la vida social. Por ejemplo, en el campo de la política hay cada vez más la participación de las mujeres. En Brasil eso es un dato significativo:

\section{Participación de las mujeres en la elección de Brasil}

\begin{tabular}{|l|c|c|}
\cline { 2 - 3 } \multicolumn{1}{l|}{ Tabla 2.1.1 } & 1998 & 2002 \\
\hline Gobernador & 1 & 5 \\
\hline Senador & 5 & 8 \\
\hline Disputado Federal & 29 & 42 \\
\hline Disputado Estadual & 107 & 129 \\
\hline Alcalde & 0 & 5 \\
\hline
\end{tabular}

Observaciones:

$318(6 \%)$ Alcaldes y $6,992(12 \%)$ legisladores municipales son mujeres;

$33 \%$ de los magistrados en el Sistema Federal de Justicia Brasileña fusticia comin y del trabajoj son mujeres

Las mujeres están a cada año ejerciendo más y más cargos políticos en Brasil lo que significa que ellas están conquistando más espacio también en otros sectores de la sociedad, como es el caso de la ciencia y tecnología. Los

9 http://www.vdi.de/studie/daten $-4 . h$ tml 
datos sobre la participación femenina en la investigación científica y tecnológica evidencian esa afirmación (gráfico 2.1.1 y tabla 2.1.2).

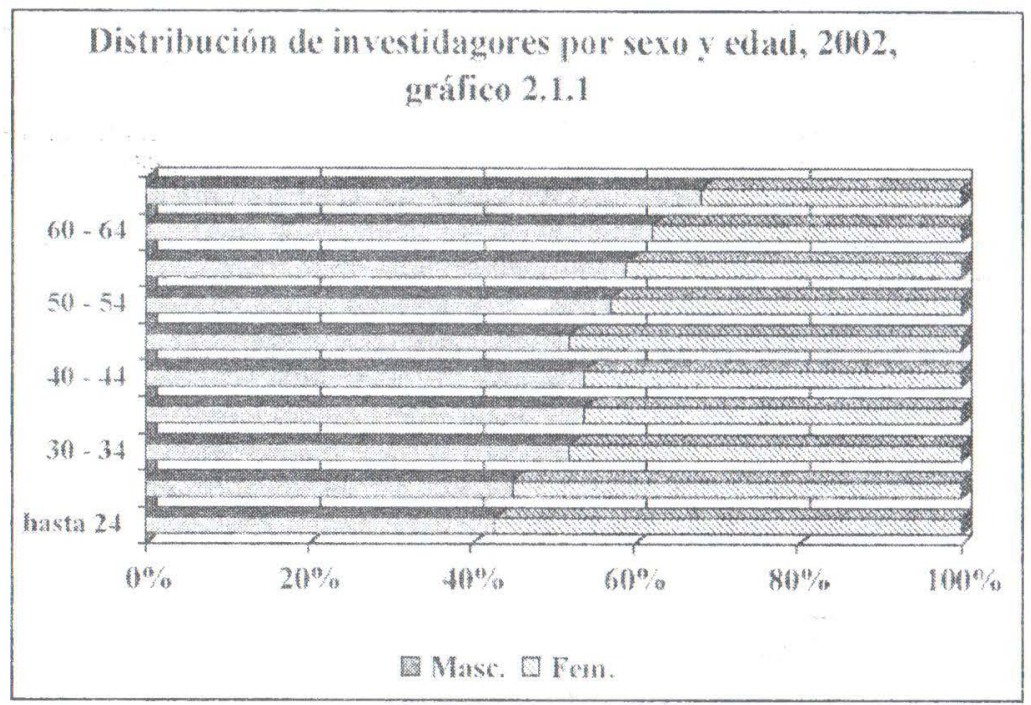

Distribución de investigadores por sexo y edad, 2002,

Tabla 2.1.2

\begin{tabular}{|c|c|c|c|c|c|}
\hline \multirow{2}{*}{ Edad en año } & Total & \multicolumn{2}{|c|}{ Número de } & \multicolumn{2}{c|}{ Porcentaje de } \\
\cline { 3 - 6 } & & hombres & mujeres & hombres & mujeres \\
\hline Hasta 24 & 303 & 131 & 172 & 43,2 & 56,8 \\
\hline $25-29$ & 2642 & 1187 & 1453 & 45,0 & 55,0 \\
\hline $30-34$ & 6250 & 3235 & 3014 & 51,8 & 48,2 \\
\hline $35-39$ & 10572 & 5680 & 4891 & 53,7 & 46,3 \\
\hline $40-44$ & 10510 & 5626 & 4881 & 53,5 & 46,5 \\
\hline $45-49$ & 10161 & 55305 & 4885 & 52.2 & 47,8 \\
\hline $50-54$ & 8222 & 4648 & 3574 & 56,5 & 43,5 \\
\hline $55-59$ & 4674 & 2754 & 1917 & 59,0 & 41,0 \\
\hline $60-64$ & 2172 & 1353 & 819 & 62,3, & 37,7 \\
\hline 65 y más & 133 & 903 & 429 & 67,8 & 32,2 \\
\hline Total & 56891 & 30859 & 26021 & 54,3 & 45,7 \\
\hline
\end{tabular}


Cuanto más jovenes son las mujeres más grande es su participación en los financiamentos para investigaciones científicas y tecnológicas (tabla 2.1.2). Si vamos a las edades mayores vemos que la participación masculina es más importante. Eso nos permite decir que las mujeres están conquistanto más espacio en la ciencia y tecnologia a cada año porque la diferencia de percentuales entre la distribución masculina y femenina disminuye según la edad hasta la cantidad de mujeres con menos que treinta anõs superar la de hombres.

Con relación a las áreas del conocimiento hay una grande diferencia entre la participación masculina y femenina en la investigatión científica y tecnológica. En la tabla 2.1.3 se puede ver que las mujeres están en más grande cantidad en las áreas de las ciencias humanas, seguidas por las ciencias de la salud, biológicas, letras y artes; la proporción de las mujeres es más grande en las ciencias de letras, seguida por la ciencias humanas y de la salud. Por otro lado, en áreas como ciencias exactas, ingeniería y computación el número de hombres es más grande, sólo un cuarto de los investigadores son mujeres.

\section{Número de investigadores por sexo y área de conocimiento}

Tabla 2.1.3

\begin{tabular}{|l|c|c|c|c|}
\hline \multirow{2}{*}{ Área de conocimiento } & \multirow{2}{*}{ Total } & \multicolumn{2}{|c|}{ Nümero de } & Porcentaje de \\
\cline { 3 - 5 } & & hombres & mujeres & mujeres \\
\hline Linguística, Literatura, Artes & 2874 & 912 & 1962 & $68,3 \%$ \\
\hline Ciencias Humanas & 10811 & 4301 & 6506 & $60,2 \%$ \\
\hline Ciencias Sociales Aplicadas & 5843 & 3075 & 2766 & $47,3 \%$ \\
\hline Ciencias Exactas & 7936 & 5493 & 2441 & $30,8 \%$ \\
\hline Ciencias Biológicas & 8191 & 3894 & 4296 & $52,5 \%$ \\
\hline Ciencias de Salud & 10408 & 4355 & 6051 & $58,1 \%$ \\
\hline Ciencias Agarias & 7611 & 5139 & 2472 & $32,5 \%$ \\
\hline Ingenería y Ciencia de la & & & & $24,5 \%$ \\
\hline Computación & 9668 & 7300 & 2368 & $\mathbf{4 5 , 6 \%}$ \\
\hline
\end{tabular}

La tabla 2.1.4 muestra el número de investigadores por titulación, sexo y área del conocimiento. Los números revelan un hecho importante: la diferencia entre la participación femenina y masculina en las áreas donde las mujeres están en más grande cantidad, como por ejemplo ciencias humanas y ciencias de la salud, es una diferencia progresivamente más pequeña según la titulatión es más alta. Luego, considerandose la titulación académica, las mujeres están en menor cantidad entre los doctores. Las diferencias entre hombres y mujeres es más pequeña en las áreas "femeninas" en el nivel de doctorado, mientras en las áreas de las ciencias exactas, ingenierías y computatión, o sea, las áreas "masculinas", esa diferencia es ignificativamente 
más grande.

Esos datos nos permiten decir que muchas mujeres inician suas carreras profisionales como investigadoras en ciencia y tecnología, pero van hasta un punto de sus vidas, después, por motivos familiares o por no suportar las presiones de la competitión (ambos los motivos están relacionados a sus posiciones de género) no siguen adelante.

\section{Número de investidagores por titulación, sexo y área del conocimiento}

Tabla 2.1.4

\begin{tabular}{|l|r|r|r|r|r|r|r|}
\hline \multirow{2}{*}{ Área de conociniento } & \multicolumn{2}{|c|}{ Doctorado } & \multicolumn{2}{c|}{ Maestria } & \multicolumn{2}{c|}{ Licenciatura } & Total \\
\cline { 2 - 8 } & Masc. & Fem. & Masc. & Fem. & Masc. & Fem. & \\
\hline Linguística, Literatura, Artes & 98 & 225 & 245 & 618 & 555 & 1065 & 2874 \\
\hline Ciencias Humanas & 442 & 970 & 1269 & 2360 & 2481 & 3023 & 10811 \\
\hline Ciencias Sociales apl. & 358 & 468 & 956 & 1065 & 1698 & 1156 & 5843 \\
\hline Ciencias Exaclas & 225 & 132 & 773 & 508 & 4454 & 17776 & 7936 \\
\hline Clencias Biológicas & 222 & 280 & 685 & 1005 & 2954 & 2980 & 8191 \\
\hline Ciencias de Salud & 470 & 954 & 814 & 1971 & 2967 & 2990 & 10408 \\
\hline Ciencias Agarias & 234 & 160 & 1238 & 766 & 3626 & 1520 & 7611 \\
\hline Ingenería y Ciencia de & & & & & & & \\
la Computación & 775 & 337 & 1616 & 706 & 4820 & 1297 & 9668 \\
\hline \multicolumn{1}{|c|}{ Total } & $\mathbf{2 8 4 2}$ & $\mathbf{3 5 2 6}$ & 7596 & $\mathbf{8 9 9 9}$ & $\mathbf{2 3 5 5 5}$ & $\mathbf{1 5 8 0 4}$ & $\mathbf{6 3 3 4 2}$ \\
\hline
\end{tabular}

\subsection{La vista especial de CEFET-PR}

El Centro Federal de Educação Tecnológica do Paraná - CEFET-PR es una escuela con más de 90 años que tuvo inicio como escuela de aprendizes y artífices, para niños de familias pobres, con el objetivo de darles una formación profesional y hoy se transformó en una escuela de enseñanza superior con cursos de nivel medio, universitario y programas de pos-grado, como maestría y doctorado. Está situada en la ciudad de Curitiba, capital del Estado del Paraná en la región sur del Brasil y tiene actualmente más 5 unidades en el interior del estado.

Esa escuela es especial para estudiar la participación de las mujeres y hombres en ciencia y tecnología por qué es la más antigua del país en educación tecnológica y se presenta como un universo muy fortemente masculino a pesar de observarse una tendencia de crecimiento de la participación de las mujeres entre alumnos y profesores.

Los datos que siguen son de los cursos técnicos del CEFET-PR Unidad de Curitiba - y muestran que la cantidad de varones es significamente más grande que el número de las mujeres en todos los cursos de la área tecnológica, excepto en el curso de Diseño Industrial. La diferencia más grande 
está en el Curso Técnico de Mecánica, donde la cantidad de mujeres es insignificante, mientras en el curso de Diseño Industrial la mayoría es de mujeres, aún que en esto último caso, la cantidad de varones es bien más grande que la de las mujeres en el Curso de Mecánica. En el curso de Edificaciones la cantidad de varones y chicas está más o menos equilibrada.

\section{Distribución de los alumnos de CEFET/PR por sexo}

Tabla 2.4.5

\begin{tabular}{|c|c|c|c|c|c|c|}
\hline \multirow[t]{2}{*}{ Especialidad } & \multicolumn{2}{|c|}{1997} & \multicolumn{2}{|c|}{1998} & \multicolumn{2}{|c|}{1999} \\
\hline & $\%$ masc. & $\%$ fem. & $\%$ masc. & $\%$ fem. & $\%$ masc. & $\%$ fem. \\
\hline Electrónica & 89 & 11 & 88 & 12 & 90 & 10 \\
\hline Electrotécnica & 86 & 14 & 87 & 13 & 90 & 10 \\
\hline Edificaciones & 55 & 45 & 55 & 45 & 55 & 45 \\
\hline Mecánica & 94 & 6 & 94 & 6 & 95 & 5 \\
\hline Telecomunicaciones & 69 & 31 & 70 & 30 & 72 & 28 \\
\hline Diseño Industrial & 35 & 65 & 35 & 65 & 36 & 64 \\
\hline Total & 73 & 27 & 73 & 27 & 75 & 25 \\
\hline
\end{tabular}

Fuente: SILVA, Nanci Stancki. Gênero e trabalho feminino: estudo sobre as representações de alunos(as) dos cursos técnicos de desenho industrial e mecânica do CEFET-PR. Dissertação de mestrado, PPGTE/CEFET-PR, 2000.

En total ni la proporción de las mujeres ni su distribución sobre las especialidades no ha cambiado en el espacio de tiempo, mas las variaciones entre los especialidades son notables.

\subsection{La vista nacional en Alemania}

Anche en Alemania la participación de las mujeres en la política se ha cambiado en las últimas decadas (grafico 2.3.1). En el primer Parlamento Aleman después la reunifición 1990 la proporción de las disputadas ascendió a $20,5 \%$. Sólo 12 aÔos después la proporción subiba a 32,3\%.

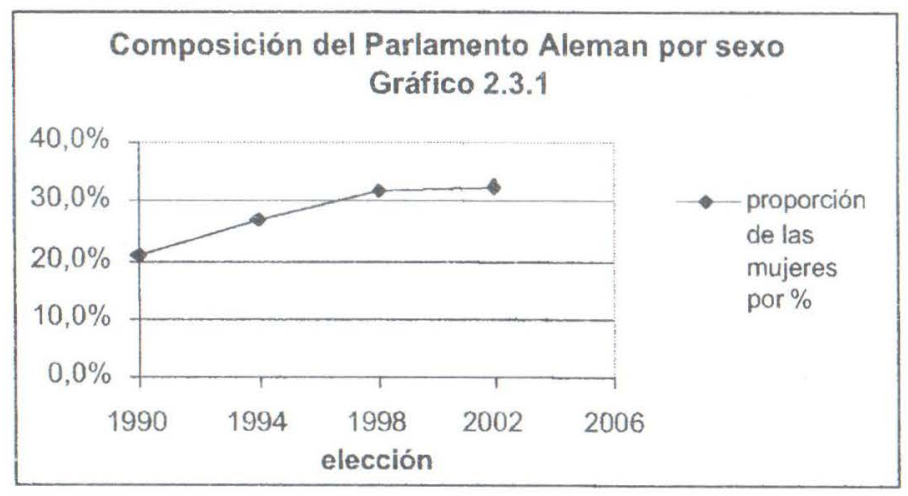


Tambien en los parlamentos estatales el número de las mujeres está aumentando. Pero las diferencias entre los estados son considerables, por ejemplo en el estado de Bremen $40,0 \%$ de los disputados del parlamento presente son mujeres, mientras que sea $21,9 \%$ en el estado de BadenWürttemberg. Nomás que un estado está goberniando por una mujer.

Simultaneamente las mujeres alemanas están al punto de conquistar las universidades y la ciencia ${ }^{1 n}$ (gráfico 2.3.2). Por la primera vez terminaban en 1994 tanto chicas como chicos la preparatoria. En 2002 matriculaban más chicas que chicos a las universidades alemanas. Casi la mitad de los licenciados y maestros, y más que un tercero de los doctorados de hoy son mujeres. Pero la proporción de las investigadoras es sólo $27,7 \%$, y de los profesores numerarios $11,9 \%$ son mujeres, si bien su numero se haya duplicado después 1992. Es que suponer que el "techo de vidrio", el glass ceiling de las universidades alemanas se encuentra entre el doctorado y la profesura. La situación en los institutos de investigación publicos es más o menos igual, allá asciendió la proporción de las mujeres investigadoras a $25,6 \%$ en $2002^{11}$.

Proporción de las mujeres en varias fases e la carrera academica, gráfico 2.2.1

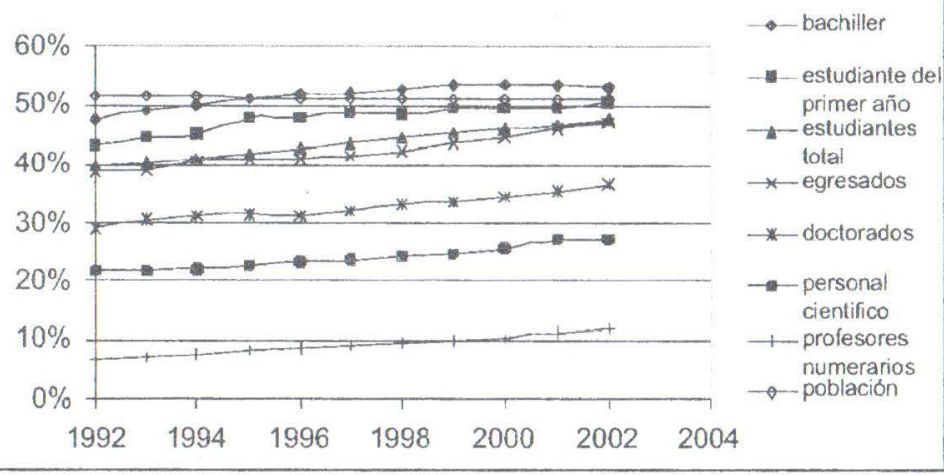

Fuente: Statistisches Bundesamt: Hochschulstandort Deutschland, 2003, Tab.18

Al interpretar el gráfico 2.3.2 tenemos que considerar un efecto del retraso: las profesoras numerarias de hoy estudiaron en un período en que la proporción de las mujeres era poca. El pequeño numero de las profesoras refleja por una parte esta situación, por la otra es debido también a las varias barreras de la carrera femenina.

${ }^{10}$ Statistisches Bundesamt: Hochschulstandort Deutschiand 2003, Wiesbaden 2003, Tab.18

11 Bund-Länder-Kommission: Frauen in Führungspositionen an Hochschulen und außerhochschulischen Forschungseinrichtungen. Heft 109, 2003, Tab. 10.1 
¿Es logrado en principió la equiparación de la mujer en las universidades alemanas? ¿Será en breve la mitad del cielo académico de las mujeres? ¿ Ponemos esperar relajadamente hasta el momento, quando las mujeres cuben la mitad de las cátedras? Una mirada a la distribución de las áreas de estudio y de investigaciónes por sexo nos sirve de escarmiento.

Las investigadoras alemanas se concentran en áreas tipicamente femeninas como las ciencias de letras, idiomas, cultura, como medicina o arte (tabla 2.3.1). Ellas rehuyen áreas como ingenería y ciencias exactas, así llamadas áreas masculinas. Más que la mitad de las investigadoras trabajan en las ciencias humanas y la medicina. Esta tendencia es bien conocida de un gran numero de paises y refleja una comprensión tradicional de los roles de las mujeres y los hombres. La distribución por áreas en Brasil (tabla 2.1.3) es más o menos parecido con Alemania, si tenemos en cuenta que la proporción de las investigadoras en Brasil con $45,6 \%$ cerca dobla la proporción en Alemania con $27,0 \%$.

\section{Investigadores en universidades alemanas por especialidad y sexo, 2001}

Tabla 2.3.1

\begin{tabular}{|c|c|c|c|}
\hline \multirow{2}{*}{ Especialidad } & \multicolumn{2}{|c|}{ Numero de } & \multirow{2}{*}{$\begin{array}{c}\text { Porcentaje de } \\
\text { mujeres }\end{array}$} \\
\hline & mujeres & hombres & \\
\hline Ciencias de letras, idiomas, cultura, deportivo & 8.257 & 13.233 & $38,4 \%$ \\
\hline Jurisprudencia, economia, ciencias sociales & 4.783 & 14.666 & $24,6 \%$ \\
\hline Matematica, ciencias exactas, informatica & 6.940 & 28.598 & $19,5 \%$ \\
\hline Medicina & 14.492 & 26.415 & $35,4 \%$ \\
\hline Agronomía, silvicultura, trofología & 1.175 & 2.775 & $29,7 \%$ \\
\hline Ingeneria & 3.143 & 22.759 & $12,1 \%$ \\
\hline Arte & 1.566 & 3.743 & $29,5 \%$ \\
\hline Total & 43.228 & 117.161 & $27,0 \%$ \\
\hline
\end{tabular}

Fuente: Statistisches Bundesamt; Fachserie 11, Reihe 4.4. De: GEW-Gender-Report 2003: Daten zur Entwicklung in Bildung und Wissenschaft

Desgraciadamente falta una estatistica sobre las investigadores por especialidad y edad en Alemania. Como indicador del desarrollo temporal de la participación academica de las mujeres en las diversas áreas academicas usamos los examenes academicos de diferentes niveles en 2001 (tabla 2.3.2). Por término medio un(a) estudiante necesita cerca 10 años del primer semestre hasta terminar el doctorado. Al analizar la tabla 2.3.2 sobre los examines aprobados tres observaciones destacan en cuanto a la selección de la especialidad por sexo:

Enseñar a las escuelas alemanas es una profesion femenina, ? de los egresados son mujeres,

Al elegir los estudios universitarios los chicos siguen también en el año 2001 roles tradicionales: las mujeres prefieren ciencias humanas 0 arte, 
los hombres ingenería, informática y ciencias exactas; el desarrollo de la última década no aporta un indicio de un cambio,

En el estudio para ser profesora de enseñanza la proporción de las mujeres en la especialidad matemática, ciencias exactas y informática ascende a ?, mientras que por la maestría la proporción de las mujeres es sólo?. Evidentamente en combinación con una profesión femenina como profesora los conceptos de ser una mujer y ser una matemática se vuelven compatibles, mientras que la ingeneria mantenga inatractiva por las mujeres tanto la carrera docente como la carrera industrial.

\section{Participación de las mujeres y los hombres de examines aprobados por especialicación y cualificación porcentualmente, 2001}

Tabla 2.3.2

\begin{tabular}{|c|c|c|c|c|c|c|c|c|c|c|}
\hline \multirow[t]{2}{*}{ Especlalldad } & \multicolumn{2}{|c|}{ Doctorado\% } & \multicolumn{2}{|c|}{ Maestria \% } & \multicolumn{2}{|c|}{ 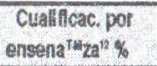 } & \multicolumn{2}{|c|}{$\begin{array}{c}\text { Ucenclatura } \% \\
\%\end{array}$} & \multicolumn{2}{|c|}{$\begin{array}{l}\text { Estudiantes del } \\
\text { primer sem. \% }\end{array}$} \\
\hline & Muj: & Homb. & Mui. & Homb. & $\mathrm{Mu}$. & Homb. & Mui. & Homb. & Muti. & Homb. \\
\hline $\begin{array}{l}\text { Ciencias de letras, } \\
\text { diomas, cultura }\end{array}$ & 45,3 & 54,7 & 69,7 & 30,3 & 77,9 & 22,1 & 73,7 & 26,3 & 65,7 & 34,3 \\
\hline $\begin{array}{l}\text { Jurispr., economia, } \\
\text { ciencias socialos }\end{array}$ & 29,3 & 70,7 & 42,2 & 57,8 & 65,4 & 34,6 & 50,7 & 49,3 & 45,7 & 54,3 \\
\hline $\begin{array}{l}\text { Matematica, ciencias } \\
\text { exactas, informática }\end{array}$ & 27,8 & 72,2 & 37,8 & 62,2 & 61,9 & 38,1 & 18,3 & 81,7 & 34,8 & 65,2 \\
\hline Medicina & 45,2 & 54,8 & 50,0 & 50,0 & - & - & .14 & - & 53,1 & 46,9 \\
\hline Veterinaria & 69,5 & 30,5 & 79,9 & 20,3 & - & - & - & - & 80,4 & 19,6 \\
\hline $\begin{array}{l}\text { Agronomia, silvicult, } \\
\text { trofologia }\end{array}$ & 33,5 & 66,5 & 56,3 & 51,8 & 87,6 & 12,4 & 51,8 & 48,2 & 53,7 & 46,3 \\
\hline ingenería & 11,4 & 78,6 & 21,1 & 19,8 & 22,9 & 77,1 & 19,8 & 80,2 & 20,5 & 79,5 \\
\hline Arte & 59,1 & 40,9 & 60,2 & 61,6 & 77,6 & 22,4 & 61,6 & 38,4 & 62,7 & 37,3 \\
\hline Total & 35,3 & 64,7 & 51,5 & 48,5 & 64,3 & 35,7 & 46,0 & 54,0 & 46,1 & 53,9 \\
\hline
\end{tabular}

Fuente: Statistisches Bundesamt; Fachserie 11, Reihe 4.3.1. De: GEW-Gender-Report 2003: Daten zur Entwicklung in Bildung und Wissenschaft

Dentro de las especialidades las mujeres no se distribuyen homogeneamente por los subgrupos. Por ejemplo, en el año 2000 ascendió la proporción de las egresadas en la informática en total a 10\%, particularmente en la informática de ingenería ascendió a $4,2 \%$ y en la informática de medios a $19,4 \%{ }^{15}$.

12 en Alemania existen examenes especiales por ser profesores de la primaria, secundaria y preparatoria.

13 la licenciatura es igualada con la diploma de las escuelas de ciencia aplicada (Fachhochschulen),

${ }^{14}$ en medicina y veterinaria no existe elnivel de la licenciatura en Alemania.

${ }^{15}$ Bund-Länder-Kommission: Frauen in den ingenieur- und naturwissenschaftichen Studiengängen. Heft 100 , tab.5.2.1 
Diferencias entre hombres y mujeres observamos tambien después terminar los estudios academicos. Las estatisticas nacionales muestran una asimetria del empleo entre mujeres y hombres en Alemania (grafico 2.3.3). Generalmente, el riesgo del desempleo es más grande por las mujeres que por los hombres. En 1995 la tasa del desempleo entre los academicos alemanos acendió a $5,4 \%$ pgr las mujeres y a $3,8 \%$ por los hombres ${ }^{16}$. Las variaciones entre las disciplinas son notables y no en correlación con la proporción de las mujeres en la disciplina. En las ciencias de letras, idiomas y cultura la probabilidad del desempleo es "sólo" $40 \%$ más alta para las mujeres que los hombres, pero en las otras disicplinas la probabilidad del desempleo es más o menos el doble para las mujeres, o sea ingenería $(90 \%)$, medicina $(110 \%)$, ciencias exactas $(90 \%)$ o jurisprudencia y economía $(80 \%)$. La FHF no dispone de una estatistica sobre el empleo o desempleo de sus egresados despues el examen. Sapememos por experiencia que las mujeres necesitan más tiempo y un número de solicitudes más grande antes de que encuentran un empleo.

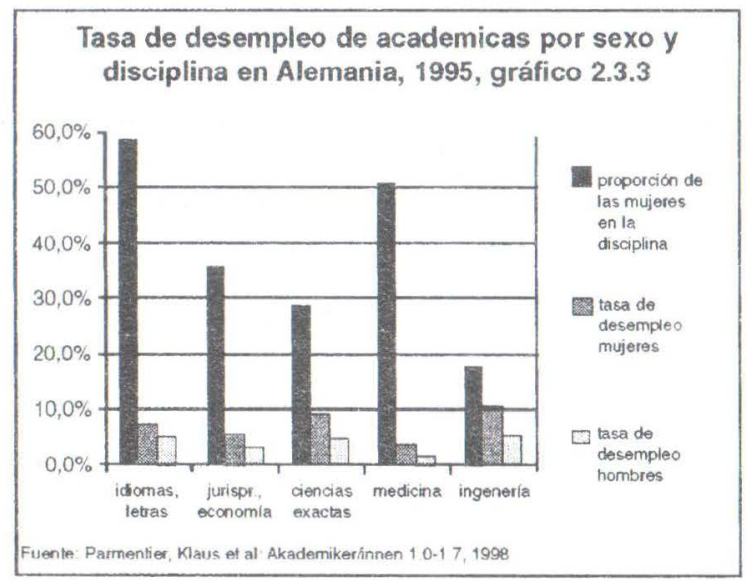

La pregunta, que hace la política como la ciencia es iporqué las mujeres no se interesan tan poco por los estudios de ingeneria, informática o ciencias exactas pese a toda la publicidad del gobierno y organisaciones diversas? ¿Porque rehuyen las mujeres especialmente las partes central de la ingenería y informática como técnica de producción allí, donde se desarrolla las tecnologías futuras en Brasil asi como en Alemania? Vamos a acercar a estas preguntas hacía la perspectiva de la educación de las escuelas técnologicas superiores.

${ }^{11}$ Fuente: Klaus Parmentier et al: MatAB, 1.0-1.7, Sonderserie ,Akademiker/innen- Studium und Arbeitsmark t", Nürnberg, 1998 


\subsection{La vista especial de la FHF}

Furtwangen es una ciudad pequeña en el sur-oeste de Alemania, en la serranía de la Selva Negra. La Escuela Técnica Superior de Furtwangen (Fachhochschule Furtwangen con el acrónimo FHF), fundada en 1852 es una de las escuelas técnicas superiores más pequeñas y más viejas del pais. Sus facultades de informática ocupan el quinto puesto entre las universidades de Alemania.

Con el comienzo del año 2002 se fundó el Centro de Competencias TanGenS - Technology and Gender in Applied Sciences - a la FHF. En este centro debe concentrarse y instituzionalisarse las actividades sobre género de la universidad. En el semestre de inverno 2002/3 el centro ha arrancado una encuesta extenso de sus estudiantes - mujeres y hombres- sobre la actitud con respecto a la tecnologia, el estudio, la carrera y el proyecto de vida. Los resultados detallados son publicados como informe de trabajo 1 y 2 de TanGenS ${ }^{17}$ o se puede descargar del homepage de TanGenS en el internet ${ }^{18}$.

\section{La proporción de los sexos por facultad y especialidad en la FHF}

Como en todos las universidades técnicas alemanas la majoría de sus integrantes son hombres. Pero en comparación con otras universidades técnicas alemanas la proporción de las mujeres es considerable. Ella ascende a $25,6 \%$ por los estudiantes y a $7,5 \%$ por los profesores numerarios. Como en el caso del CEFET (tabla 2.2.1) las mujeres y los hombres se distribuyen bastante heterogeneamente sobre las disciplinas (tabla 2.4.1) y las especialidades (tabla 2.4.2). En economía las mujeres prevalecen claramente, en la ingenería la proporción de las mujeres, que forman casi un tercero de los estudiantes es insolitamente alta en un escala alemana, mientras que en la informática las mujeres sean un minoridad con $14,8 \%$.

Vista panoramica de la proporción de las mujeres y los hombres por disciplina en 2002

Tabla 2.4.1

\begin{tabular}{|c|c|c|c|c|}
\hline \multirow{2}{*}{ Disciplina } & \multicolumn{2}{|c|}{ Número do } & \multicolumn{2}{|c|}{ Porcentaje } \\
\hline & mujeres & hombres & mujeres & hombres \\
\hline Informática & 248 & 1422 & $14,8 \%$ & $85,2 \%$ \\
\hline Ingenería & 292 & 703 & $29,3 \%$ & $71,7 \%$ \\
\hline Economía & 289 & 150 & $65,8 \%$ & $34,2 \%$ \\
\hline Total & 829 & 2415 & $25,6 \%$ & $74,4 \%$ \\
\hline
\end{tabular}

Fuente : Rectoría de la Escuela Tecnológica Superior (FHF), 2003

Vale la pena analizar la distrubución en más detalle (tabla 2.4.2). Como en el caso del CEFET-PR los estudiantes femeninos rehuyen especialidades, que se contran entre "el corazon" de la tecnica. Donde se ubican especialidades como Microsystems Engineering ( $0 \%$ mujeres), Computer Networking (2,4\% mujeres), Computer or Electrical Engineering ( $4,8 \%$ mujeres 
cada), Técnica deAutomatización (4,6\% mujeres), Informática General $(7,4 \%$ mujeres) and Precision Engineering (8,6\% mujeres).

Por todas las tecnologías en relación con la medicina, el medio ambiente, la microbiología o la alimentación la proporción de las mujeres se dispararon, por ejemplo por biotecnología (60,2\% mujeres), Tecnología Ecológica (38,1\% mujeres) o Biomedical Engineering (27,6\% mujeres). Asimismo chicas se sienten atraidas por tecnologías en relación con diseño como Informática de Medios (25,7\% mujeres) o Documentación y Diseño (50,9\% mujeres), o tecnología en relación con comunicación humano como por el caso de Business Consulting (27,3\% mujeres). Generalmente podemos concluir, que a las chicas no interesa tanto la tecnologica por la tecnología misma quanto su aplicación. Más detalles sobre la posición de 1@s chic@s frente la tecnología, sus estudios y género se entran mas adelante.

Estudiantes en la FHF en el semestre de inverno 2002/3 por sexo, facultad y especialidad, tabla 2.4.2

\begin{tabular}{|c|c|c|c|c|c|c|}
\hline \multirow[t]{2}{*}{ Facultades y especialldades } & \multicolumn{2}{|c|}{ Nivel } & \multicolumn{2}{|c|}{ Número } & \multicolumn{2}{|c|}{ Porcentaje } \\
\hline & Lic. & Maestr. & mujeres & hombres & mujeres & hombres \\
\hline \multicolumn{7}{|l|}{ Computer\&Electrical Engineering } \\
\hline Microsystems Engineering & & $x$ & 0 & 41 & $0 \%$ & $100 \%$ \\
\hline Computer Engineering & $x$ & & 6 & 120 & $4,8 \%$ & $95,2 \%$ \\
\hline Electrical Engineering & $\mathrm{x}$ & & 5 & 100 & $4,8 \%$ & $95,2 \%$ \\
\hline Subtotal & & & 11 & 261 & $4,0 \%$ & $96,0 \%$ \\
\hline \multicolumn{7}{|l|}{ Digltal Media } \\
\hline Computer Science in Media & & $x$ & 5 & 17 & $22,7 \%$ & $77,3 \%$ \\
\hline Informática de Medios & $x$ & $x$ & 83 & 240 & $25,7 \%$ & $74,3 \%$ \\
\hline Online.medios & $x$ & & 22 & 76 & $22,4 \%$ & $77,6 \%$ \\
\hline Total & & & 110 & 333 & $24,8 \%$ & $75,2 \%$ \\
\hline \multicolumn{7}{|l|}{ Informática } \\
\hline Informática General & $x$ & & 15 & 187 & $7,4 \%$ & $92,6 \%$ \\
\hline Computer Networking & $\mathrm{x}$ & & 7 & 277 & $2,5 \%$ & $97,5 \%$ \\
\hline Subtotal & & & 22 & 464 & $4,5 \%$ & $95,5 \%$ \\
\hline \multicolumn{7}{|l|}{ Informática en economia } \\
\hline Business Consulling & & $x$ & 12 & 32 & $27.3 \%$ & $72.7 \%$ \\
\hline Informática en Economía & $\mathrm{x}$ & & 61 & 312 & $16,4 \%$ & $83,6 \%$ \\
\hline Business Networking (?)? & $x$ & & 37 & - & $100 \%$ & \\
\hline Subtotal & & & 110 & 344 & $24,2 \%$ & $75,8 \%$ \\
\hline \multicolumn{7}{|c|}{ Mechanical \& Process Engineering } \\
\hline Biomedical Engineering & & $x$. & 8 & 21 & $27.6 \%$ & $72.4 \%$ \\
\hline Biotecnologia & $x$ & & 53 & 35 & $60,2 \%$ & $39,8 \%$ \\
\hline Precision Engineering & $x$ & & 3 & 32 & $8.6 \%$ & $91,4 \%$ \\
\hline Tecnología de Automatización & $\bar{x}$ & & 8 & 165 & $4,6 \%$ & $95,4 \%$ \\
\hline Tecnología Ecológica & $x$ & & 45 & 73 & $38,1 \%$ & $61,9 \%$ \\
\hline Subtotal & & & 117 & 326 & $26,4 \%$ & $73,6 \%$ \\
\hline \multicolumn{7}{|l|}{ Product Engineering } \\
\hline Documentación y Diseño & $x$ & & 57 & 55 & $50,9 \%$ & $49,1 \%$ \\
\hline Marketing and Sales & $x$ & & 35 & 138 & $20,2 \%$ & $79,8 \%$ \\
\hline Product Engineering & $x$ & & 17 & 112 & $13,2 \%$ & $86,8 \%$ \\
\hline Subtotal & & & 109 & 305 & $26,3 \%$ & $73,7 \%$ \\
\hline \multicolumn{7}{|l|}{ Economía } \\
\hline International Business Management & & $\mathrm{x}$ & 11 & 15 & $42,3 \%$ & $57,7 \%$ \\
\hline International Business Management & $x$ & $x$ & 51 & 43 & $54,3 \%$ & $45,7 \%$ \\
\hline Ciencias empresariales internacionales & $x$ & 227 & 92 & $71,2 \%$ & $28,8 \%$ & \\
\hline Subtotal & & & 289 & 150 & $65,8 \%$ & $34,2 \%$ \\
\hline Total & & & 829 & 2415 & $25.6 \%$ & $74.4 \%$ \\
\hline
\end{tabular}

Fuente : Rectoría de la Escuela Tecnológica Superior (FHF) 2003 
En los ultimos años no se ha cambiado significantamente la proporción de las mujeres en el nivel de las especialidades, por exemplo en presicion engineering la proporcion de las mujeres queda con $4 \%$ despues hace 10 años, así como en tecnología ecológica la proporción es siempre más que $40 \%$. Correspondiente del ciclo de la coyuntura, de la situación en el mercado de trabajo y también de "modas" al eligir su carrera academica, fluctua el número de los estudiantes por especialidad y en consecuencia la proporción de las mujeres en la universidad. Por ejemplo debido a la ampliación de la facultad Digital Media, una facultad con un porcentaje de las mujeres de $25 \%$, aumenta la proporción de las mujeres en toda la universidad.

\section{PORQUÉ ESTUDIAR INGENERÍA O INFORMATICA?}

La pregunta que conmueve la politica, la economía y las universidades dice en el fondo, porqué las mujeres no estudian ingenería o informática? Porque queja la proproción de las mujeres tan pequeño en el corazon de la tecnología? Puesto que no es posible interrogar la gran parte de las mujeres, que se decidieron en contra un estudio de tecnología, inquirimos las mujures al CEFET y a la FHF, que se decidieron por un estudio tal. Las investigaciones se diseñaron y se llevaron a cabo independientemente en los dos instituciones de educación superior, por eso no deberiamos comparar los resultados mediante las cifras, sino tambien mediante el sentido.

\section{La cuenta en el CEFET-PR}

El Curso de Ingeniería de Producción Civil del CEFET-PR presenta, año a año un número más grande de mujeres. Lo que se percibe es que ellas tienen un desempeño academico mejor que lo de los varones, como por ejemplo, en la asiduidad, los resultados de las evaluaciones y participación en las actividades de las classes. Pero muchas de ellas no están bien adaptadas en su profesión ni encuentran colocación en el mercado de trabajo, adonde la preferencia es para los hombres. Por lo tanto, a pesar del conocimiento adquirido en sus cursos, las mujeres ingenieras civiles ni siempre consiguen trabajar en el area de su formación.

Esta realidad nos llevó a la siguiente indagación: cuales son das razones que llevaran esas jóvenes a eligir Ingeniería de Producción Civil, un curso adonde ellas terian que trabajar con "peões de obra", un grupo de hombres reconocidamente hostil a la participación femenina en su trabajo? Cual alegación justificaría su elección? Cual incentivo recibieran para cursar ingeniería?

Para contestar essas preguntas fue realizado durante los años 2002/2003 una investigación entre 74 alumnas de este curso del CEFET-PR. La edad aproximada de ellas es 19 a 24 anõs y están cursando del $4^{\circ}$ al $10^{\circ}$ nivel, en grande parte en el horario nocturno. Algunas de ellas, las que habian hecho el Curso Técnico de Edificaciones, ya están trabajando en su area.

El gráfico 3.1 presenta las respuestas con respecto a la motivación de las estudiantes femeninas para eligir su especialidad. Los primeros dos items se refieren a la motivación intrinsica sobre la elección de la especialidad. La 
mayoría $(65,8 \%)$ de las mujeres siente una vocación por una profesion técnica, solo $10,8 \%$ de las mujeres se han decidido por un curso técnico por falte de alternativas. Por la mitad $(50 \%, 0)$ la perspectiva de conseguir una titulación universitaria fue importante o muy importante por la elección. Entre los motivos externos destacan la influencia de los amigos $(90,5 \%)$ y la importancia limitada de la familia $(18,9 \%$ ) y los profesores sobre la eleccion del estudio. La mitad de las mujeres $(48,7 \%)$ se decidieron a causa de une expectiva buena en el mercado de trabajo por el curso y sólo $37 \%$ a causa de una expectiva de un salario bueno. Por $73 \%$ de las mujeres fue la gratuidad del curso un argumento por matricularse al CEFET.

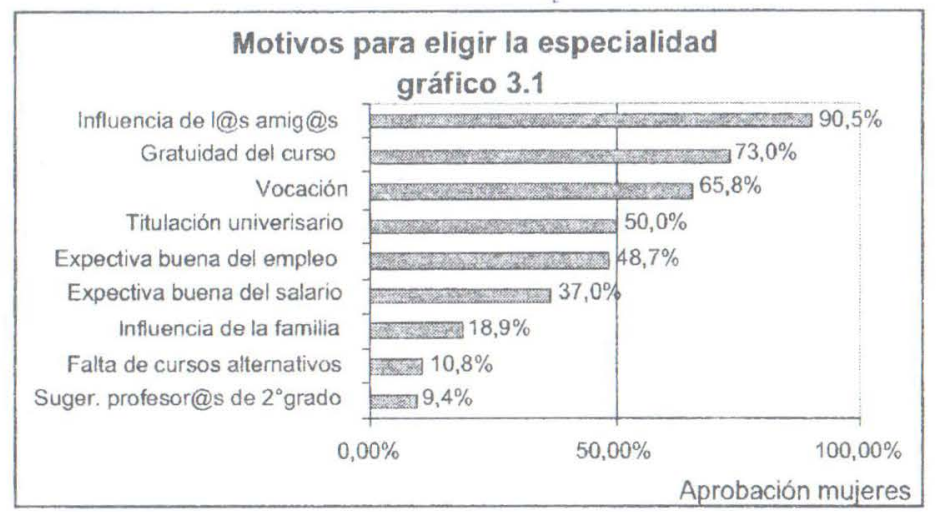

\section{La cuenta en la FHF}

Unos de los proyectos al centro TanGens, que arrancó en 2002, es dedicado al desarrollo, la implementación y evaluación de modulos didacticos para sensibilizar por género en de las especialidades selectas. El caso de Medios Digitales fue presentado en detalles al primer encuentro "Estudios de género y educación en México" a Xalapa en $2003^{19}$. Objectivo de las medidas es hacer más atractivo y ajustado los estudios a la FHF en primer lugar por las mujeres, pero también por los hombres. Para probar el efecto de los medidas para sensibilizar por género, interrogamos a los(as) estudiantes sobre sus actitudes a la técnica, sus auto-imagenes en relación a la técnica, sus expectativas hacía el empleo y la vida futura así a las posibilidades de mejorar la educación en la FHF. Usamos cuestionarios extensos y entrevistas. En la primera ola de la encuesta interrogamos con la ayuda de cuestonarios a 315 estudiantes en su primer año del estudio academico - 94 mujeres y 221

19 Munder, Irmtraud: ¿Cómo scnsibilizar cstudiantes por géncro? Medidas y resultados de acciones realizadas en la facultad de Medios Digitales de la Escuela Superior de Tecnología de Furtwangen, Alemania. En: Estudios de género y educación en México, Universidad Veracruzana, 2003 
cuales respondieron $88 \%$, y suplementario llevamos a cabo 16 entrevistas con 9 mujeres y 7 hombres. Entre los 68 juegos de preguntas del questionario selectamos aquí las que corresponden más cercanamente con ellas del cuestionario brasileño.

\section{Los motivos intrinsicos}

Las mujeres jovenes alemanas se decidieron por una carrera técnica por la expectiva del empleo y el interés (gráfico 3.2). Por $86,8 \%$ des las mujeres la oportunidad y seguridad en el puesto de trabajo es el argumento más importante en favor de una profesión técnica. Intereses especiales $(78,3 \%)$, aficiónes y talentos $(65,5 \%)$, la oportunidad de una carrera $(62,7 \%)$ así como la oportunidad de desenvolvimiento por la profesion $(59,0 \%)$ son los argumentos proximamente importantes por las mujeres. En su mayoría las repuestas de las mujeres y hombres son semejantes, si bien los hombres sienten evidentemente más interés y entusiasmo por la técnica y informática. Sólo con respecto a arreglar cosas defectuosas o la conocimientos de computadoras las mujeres y los hombres se diferencian: $55,5 \%$ de los hombres indicaron, que la alegría de arreglar las cosas defectuosas es un motivo importante para la elección de la especialidad, pero sólo 27,7\% de las mujeres; por $49,5 \%$ de los hombres su conocimientos de las compudadores es un motivo importante, pero sólo por $22,9 \%$ de las mujeres. Otras razones como la reputacion de la profesion en la sociedad o el anhelo de los padres casi no importa.

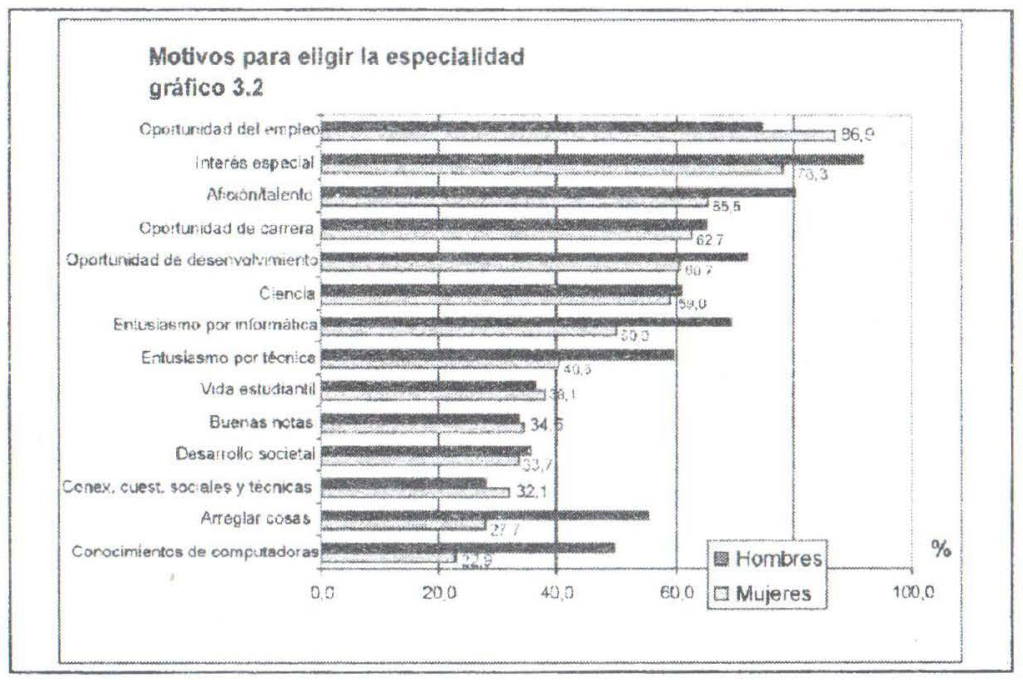

\section{Influencias extrinsicas}

Los personas que aconsejó la decisión del estudio, son representado en el grafico 3.3. Todavía los parentes ejercen un gran influencia sobre la elección 
de la especialidad de sus hijos, un poco más con las mujeres que con los hombres. $72 \%$ de las mujeres indicaron, que la madre aconsejó un estudio técnico a ella. En segundo lugar importa el consejo de las amigas $(68,4 \%$ ) y los amigos $(60,8 \%)$. Las/los hermanos, parientes y profesores tienen un papel subordinato por la decisión del estudio. Casi nadie de los personas listas desconsejó a las/los jovenes tomar una profesion técnica. A excepción de la amiga las respuestas de las mujeres y los hombres no se diferencian mucho. $68,4 \%$ de las amigas aconsejan a una mujer, $22 \%$ ni aconsejan ni desconsejan, mientras que sok, 41,3\% de las amigas aconsejan a un hombre, pero 51,5 no se pronuncian. Las mujeres piensan más frecuentamente sobre abandonar es estudio y tienden a estimar el exito de su estudio menos optimista posiblemente más realista - que los compañeros. En total las estudiantes de la FHF viven mucho respaldo de su ambito social, si se deciden por un estudio técnico. De ahi que sea dificil comprender por qué las mujeres no deciden en un número más grande por un estudio técnico y por qué las que han decidido por el sean tan escepticas con respecto al su rendimiento y la carrera profesional. Evidentemente debemos buscar las razones por esta actitud en un fase de vida antes.

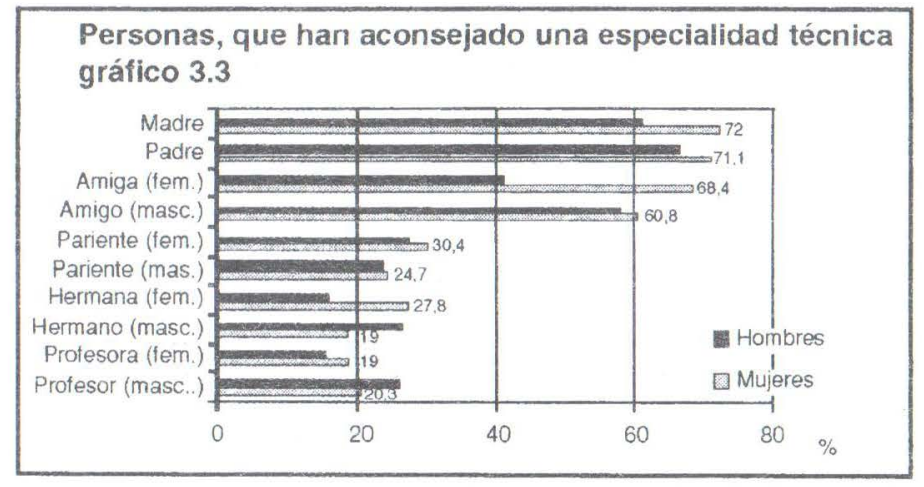

Por eso preguntamos, quien es que ha fomentado el interés en la técnica (gráfico 3.4). Con distancia la persona más importante por ambos sexos es el padre, siguen los amigos masculinos y profesores masculinos. La madre no importa mucho con respecto al interés en la técnica. Aquí entendamos mejor, por qué en Alemania la técnica tiene una connotación masculina, y aún más importante, por qué los conceptos de roles sociales, el rol de una mujer y el rol de una profesionista técnica no son facilmente compatibles. En la situación actual, marcado por la competición rigurosa en el mercado de trabajo, el deseo de reconocimiento profesional prevalece. En las entrevistas las mujeres las mujeres tienden a ignorar su género y definen la universidad y el trabajo cómo un espacio neutral, sin género. Que esta no es una solución firme por el conflicto de ser una mujer y una informática al mismo tiempo, muestra la inseguridad y desorientación profesional de las mujeres. La tensión permanente entre una personalidad individual fuerte y un auto-imagen colectiva borrosa deben ser enorme. De ahi que no sorprende que las mujeres 
no se sienten bien en sus profesiones técnicas y que unas dar las espalda a sus profesiones técnicas agotadas después algunos años de trabajo.

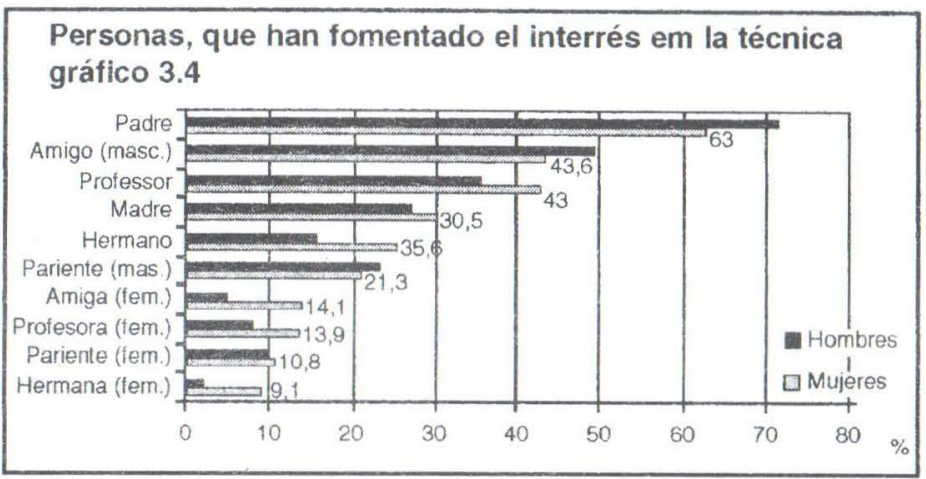

\section{INTERPRETACIÓN}

Al comparar la situación de género en tecnología y sciencia entre Alemania y Brasil prevalecen las caracteristicas comunes:

1. en ambos países las mujeres prefieren áreas como ciencias humanas o medicina,

2. si las mujeres deciden por un estudio técnico o de las ciencias exactas, prefieren life science o un estudio junto con otras áreas como medios o diseño,

3. técnica pura o ciencias exactas puras no son estudios atractivos por las mujeres,

4. en ambas universidades la proporción de los estudiantes femeninos ascende a cerca un cuarto y no se ha cambiado en los ultimos años,

5. en ambas universidades el porcentaje de las mujeres con las especialidades tecnicas diversas varian en gran escala, de 5\% hasta $64 \%$,

6. en ambas universidades las mujeres eligen un estudio técnico por afición, por la oportunidad del empleo (en Alemania más que en Brasil) y no por falta de alternativas,

7. en contraste con los estereotipos corrientes, los(as) amigos(as) y la familia son iguaimente importante en Alemania por la elección de un estudio, mientras que en Brasil domine la influencia de los(as) amigos(as) con gran distancia sobre la familia.

En Alemania como en Brasil observamos un hecho que la investigación de género respectivamente mujeres llama la simultaneidad de integración y marginalicación ${ }^{20}$ con respecto a las carreras de las mujeres altamente

${ }^{20}$ Blättcl-Mink, Birgit: Studium und Geschlecht. Faktoren einer geschlechterdifferenten Studienfachwahl in Baden-Württemberg. Arbeitsbericht der Akademie für Technikfolgenabschätzung Nr. 219, p.3, Stuttgart, 2002. 
cualificadas. Esta significa que las mujeres participan progresivamente en el sistema cientifico (integración), pero se encontran empujadas en áreas menos atractivos por los hombres (marginalización). Ulrich Beck, un sociologo aleman, escribió, que en algunas áreas las mujeres han conquistado barcas que estan en el proceso de hurviendose ${ }^{21}$, las que ya dejaron los ratones (es decir los hombres). Consiguientemente no sólo cuenta la participación de las mujeres en términos numericos - el objectivo de igualdad en números de gender mainct-ạaming ya es logrado en las universidades alemanas, en breve será logrado en Brasil - sino también o ante todo en terminos cualitativos - el objectivo debería ser la igualdad en áreas determinantes para la dinámica del proceso de la modernización, éstas son las áreas técnicas.

\section{Agradecimiento}

Las investigaciones de la FHF se han fundado de la Secretaria de Ciencis, Investigaciones y Arte del estado de Baden-Württemberg su el númer de registración $\mathrm{Az}$ 24-729.18-1-21/15.

Los datos del CNPq sobre la participación de las mujeres en investigationes científicas fueran obtenidos en el site www.cnpq.br por Juliana Schwartz, estudiante del Programa de Pós-Graduação em Tecnologia (PPGTE) do CEFET-PR. Las tablas sobre los datos fueran también elaboradas por ella a quien agradeciemos.

\footnotetext{
${ }^{21}$ Beck, Ulrich/Beck-Gemsheim, Elisabeth: Das ganz normale Chaos der Liebe. Frankfurt/Main, 1990, p.29
} 